\title{
A new construction for symmetric $(4,6)$-configurations
}

\author{
Leah Wrenn Berman * \\ Department of Mathematics \& Statistics, University of Alaska Fairbanks \\ Fairbanks, Alaska, USA \\ Nadine Alise Burtt \\ Department of Mathematics, University of Pittsburgh \\ Pittsburgh, Pennsylvania, USA
}

Received 16 November 2009, accepted 25 August 2010, published online 5 November 2010

\begin{abstract}
Geometric $(4,6)$-configurations are collections of points and straight lines, in the Euclidean plane, so that every point has four lines passing through it and every line has six points lying on it. In this paper, we present a new construction for $(4,6)$-configurations which have high degrees of geometric symmetry, by superimposing 4-astral 4-configurations with certain properties.
\end{abstract}

Keywords: Configurations, incidence geometry, symmetry.

Math. Subj. Class.: 51E30, 05B30

\section{Introduction}

A geometric $(q, k)$-configuration is a collection of points and straight lines in the Euclidean plane, so that every point lies on $q$ lines and every line passes through $k$ points; if $q=k$, we simply refer to $k$-configurations. If the number of points $p$ and lines $n$ is relevant to the discussion, we refer to a $\left(p_{q}, n_{k}\right)$ configuration. We say that a $(q, k)$-configuration is symmetric if, under rotations and reflections of the plane mapping the configuration to itself, there are fewer symmetry classes of points than the number of points in the configuration, and similarly for lines: configurations that are highly symmetric have a small number of symmetry classes of points and lines. The modern study of geometric configurations began about 20 years ago, with the discovery by Grünbaum and Rigby [13] of a

\footnotetext{
${ }^{*}$ Corresponding author.

E-mail addresses: 1wberman@alaska.edu (Leah Wrenn Berman), naburtt@gmail.com (Nadine Alise Burtt)
} 
highly symmetric drawing of a $\left(21_{4}\right)$ configuration with three symmetry classes of points and lines; since then, there has been considerable work investigating 4-configurations with various properties (see, e.g., [5, 4, 6, 1, 7, 8, 11]). However, there has been relatively little investigation of configurations where $q$ and $k$ are not equal and $[q, k] \neq[3,4]$, although there are a few results in $[3,2]$, in a very constrained situation, where the configurations have as much symmetry as possible, and there are some results in Grünbaum's recent monograph on configurations [12, Section 4.4]. The current work presents a general method of constructing $(4,6)$-configurations with four symmetry classes of lines and six symmetry classes of points, using as building blocks a reasonably well-understood class of 4-configurations called 4-astral configurations. Note that two examples of the type of $(4,6)$-configurations discussed in this paper were presented without discussion in [12, Figures 4.4.8 and 4.4.10(b)].

\section{Multiastral 4-configurations}

To construct $(4,6)$-configurations, we will use multiastral-specifically 4 -astral-4-configurations as building blocks. These configurations have been studied fairly extensively (see e.g., [4, 7, 11, 12]; in [7] they were called polycyclic and in [4, 6] they were called celestial. The current termiology is that used in Branko Grünbaum's recent monograph on configurations [12, Sections 1.5, 3.5-3.9]; the following discussion of multiastral configurations is adapted from that source as well, along with his survey article [11] and the first author's article [4].

A multiastral 4-configuration is a collection of points and straight lines in the Euclidean plane so that every point has four lines, from each of two symmetry classes, passing through it. Moreover, every symmetry class of points has the same number of points, say $m$, in it, and the points in each symmetry class form concentric regular $m$-gons. The symmetry group of the entire configuration is $d_{m}$, and every line contains two points from each of two $m$-gons. Multiastral 4-configurations are a generalization of astral 4-configurations, which are 4-configurations with precisely two symmetry classes of points and two symmetry classes of lines (see, e.g., [1, 12, 10,9]). A 3-astral configuration is shown in Figure 1 .

Multiastral configurations with $h$ symmetry classes of points and lines are called $h$ astral, and every $h$-astral configuration may be described by a configuration symbol of the form

$$
m \#\left(s_{1}, t_{1} ; s_{2}, t_{2} ; \ldots ; s_{h}, t_{h}\right),
$$

where there are $m$ points in each symmetry class of points and $m$ lines in each symmetry class of lines. Using a configuration symbol, it is possible to construct a configuration uniquely (although several different configuration symbols may correspond to the same geometric configuration, depending on a choice of labelling). In order for a configuration symbol to be valid, it must satisfy four axioms (see [11, Section 3.5] for details).

Axiom 1: $\sum_{i=1}^{h} s_{i}+t_{i}$ is even

Axiom 2: $s_{i} \neq t_{i} \neq s_{i+1}$ for $i=1, \ldots, h-1$ and, additionally, $s_{h} \neq t_{h} \neq s_{1}$

Axiom 3: $\prod \cos \left(s_{i} \pi / m\right)=\prod \cos \left(t_{i} \pi / m\right)$ 


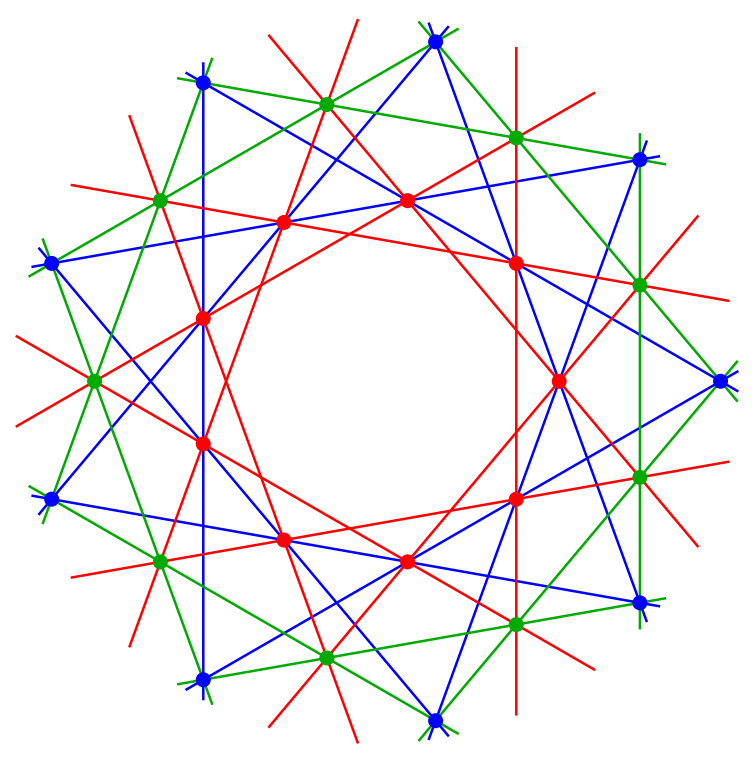

Figure 1: The 3 -astral 4-configuration $9 \#(3,1 ; 2,3 ; 1,2)$. Lines $L_{0}$ and points $v_{0}$ are blue, lines $L_{1}$ and points $v_{1}$ are red, and lines $L_{2}$ and points $v_{2}$ are green.

Axiom 4: No proper subsequence $\left(s_{i}, t_{i}, s_{i+1}, t_{i+1}, \ldots, s_{j}\right)$ generates a valid configuration symbol

$$
m \#\left(s_{i}, t_{i} ; s_{i+1}, t_{i+1} ; \ldots ; s_{j}, t *\right)
$$

that satisfies Axioms $1-3$, where $1 \leq t *<\frac{m}{2}$.

A set of lines $\ell=\left\{\ell_{0}, \ell_{1}, \ldots, \ell_{m-1}\right\}$ is of span $s$ with respect to a set of vertices $\left\{v_{0}, v_{1}, \ldots, v_{m-1}\right\}$ forming a regular $m$-gon if $\ell_{i}$ connects $v_{i}$ and $v_{i+s}$, with indices taken modulo $m$. Given a set of lines $\ell$ of $\operatorname{span} s$, the $t$-th intersections of those lines, collectively labelled $(s / / t)$ (also referred to as $[[s, t]]$ in, e.g., [4, 6]), is found by starting at the "midpoint" of $\ell_{0}$ (that is, the foot of the perpendicular line to $\ell_{0}$ that passes through the center of the configuration) and counting leftward through $t$ intersections of the lines $\ell$ with each other; see Figure 2. Given an $m$-gon and a set of lines of span $s$, allowable values for $t$ are integers from 1 to $\frac{m}{2}$.

Given a valid configuration symbol $\mathcal{C}=m \#\left(s_{1}, t_{1} ; s_{2}, t_{2} ; \ldots ; s_{h}, t_{h}\right)$, the construction method to produce the configuration is as follows (adapted from the algorithm in [4]).

Step 1: Begin with $m$ points forming the vertices of a regular $m$-gon. Collectively, these vertices will be referred to as $\left(v_{\mathcal{C}}\right)_{0}$. Typically, these vertices have coordinates $\left(\cos \left(\frac{2 \pi i}{m}\right), \sin \left(\frac{2 \pi i}{m}\right)\right)$ for $i=0,1, \ldots, m-1$.

Step 2: Construct lines collectively known as $\left(L_{\mathcal{C}}\right)_{0}$ of span $s_{1}$ that connect these vertices.

Step 3: Construct the $t_{1}$-st intersections of the lines $\left(L_{\mathcal{C}}\right)_{0}$ and call them $\left(v_{\mathcal{C}}\right)_{1}$; they have symbol $\left(s_{1} / / t_{1}\right)$. 


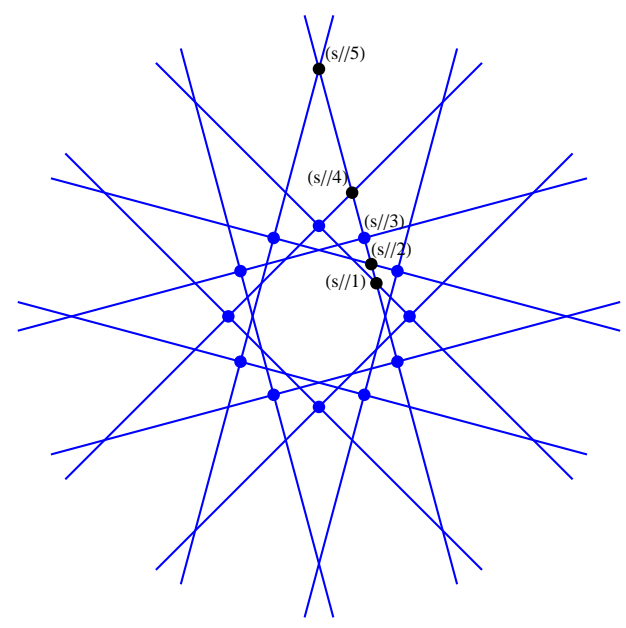

Figure 2: Lines of span $s$ with respect to points $v_{0}$ and other intersections of the span $s$ lines, labelled $(s / / t)$. Here, $m=12, s=3$ and $t=1,2,3,4,5$. The points $v_{0}$ are shown in blue.

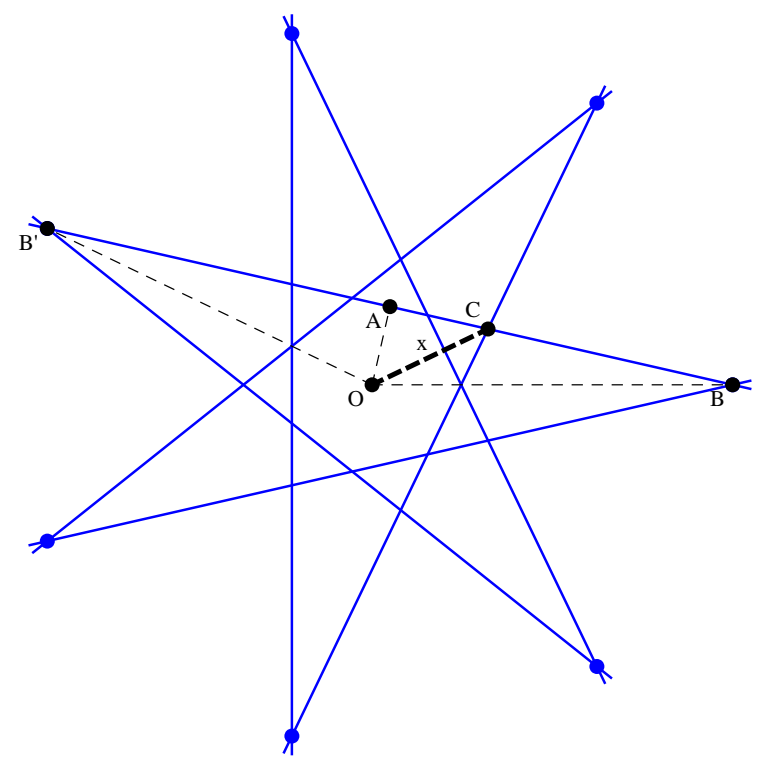

Figure 3: Determining the radius of a point with label $C=(s / / t)$ with respect to a regular convex $m$-gon with radius $r$. Since the blue lines are of span $s$, point $B^{\prime}$ has coordinates $(\cos (2 s \pi / m), \sin (2 s \pi / m))$, so $\angle B O A=\frac{s \pi}{m}$, where $A$ is the foot of the perpendicular from the center $O$ to the line $B B^{\prime}$. If $O B=r$, then since $\cos (\angle A O B)=\frac{O A}{O B}$, it follows that $O A=r \cos (s \pi / m)$. Since $C$, which has label $(s / / t)$, is the $t$-th intersection of the span $s$ lines, $\angle A O C=\frac{t \pi}{m}$. Therefore, $\cos (\angle A O C)=\frac{O A}{O C}$, so $O C=r \cdot \frac{\cos (s \pi / m)}{\cos (t \pi / m)}$. In the diagram, $m=7, s=3$ and $t=2$. 
Step 4: For $i=1, \ldots, h-1$, iteratively repeat the previous two steps: using vertices $\left(v_{\mathcal{C}}\right)_{i}$, construct lines $L_{i}$ of span $s_{i+1}$, and let $\left(v_{\mathcal{C}}\right)_{i+1}$ be the $t_{i+1}$-st intersections of the lines $L_{i}$, with label $\left(s_{i+1} / / t_{i+1}\right)$.

Because the symbol is valid, the points $\left(v_{\mathcal{C}}\right)_{h}$ must coincide with the original $m$ points labelled $\left(v_{\mathcal{C}}\right)_{0}$. The $\mathcal{C}$ subscripts may be dropped when the configuration that $v_{i}$ and $L_{i}$ refer to is either unambiguous or irrelevant.

Adapting the discussion in [6], we say that a ray of an $h$-astral configuration is a diametral ray of the configuration if it emanates from the center of symmetry of the configuration (conventionally taken to be the origin) and passes through a point from the set $v_{0}$. A ray is a mid-diametral ray if it is the rotation by an angle of $\frac{\pi}{m}$ of some diameter. If diametral rays can pass through a class of points, that class is said to be diametral or of type $D$, and likewise if mid-diametral rays can pass through the points they are said to be mid-diametral or of type $M D$. (In a typical configuration centered at the origin with one vertex in $v_{0}$ located at $(1,0)$, diametral points have angle $\frac{\pi i}{m}$ with $i$ even, while mid-diametral points have angle $\frac{\pi i}{m}$ for odd $i$.) If there are two classes of points and they both are diametral or both are mid-diametral, the classes of points are the same type. In Figure 1, the points $v_{0}$ and $v_{1}$ (blue and red) are type $D$, and thus of the same type, while the points $v_{2}$ are type $M D$.

In our construction of $(4,6)$-configurations, it is useful to be able to determine the radius of the circumcircles passing through the regular $m$-gons formed by the vertices $v_{i}$ (the "radius of the $v_{i}$ "). Using elementary trigonometry, the radius of a point with label $(s / / t)$ with respect to a regular convex $m$-gon of radius $r$ is

$$
r \cdot \frac{\cos \left(\frac{s \pi}{m}\right)}{\cos \left(\frac{t_{i} \pi}{m}\right)}
$$

see Figure 3.

Let $r_{i}$ be the radius of vertices with label $v_{i}$, and suppose $r_{0}=1$. Because of the iterative nature of the construction of $h$-astral configurations, the radius

$$
r_{j}=\prod_{i=1}^{j} \frac{\cos \left(\frac{s_{i} \pi}{m}\right)}{\cos \left(\frac{t_{i} \pi}{m}\right)} .
$$

Note that we take Axiom 3 as a necessary condition for the existence of a valid configuration because of the convention that $r_{0}=1$, the requirement that $v_{0}=v_{h}$, and using this value for $r_{h}$.

Often, $h$-astral configurations are classified by considering the cohort symbol $m \# S ; T$, where $S=\left\{s_{1}, \ldots, s_{h}\right\}$ and $T=\left\{t_{1}, \ldots, t_{h}\right\}$. Trivial $h$-astral configurations are those where $S=T$ (as sets); Axioms 1 and 3 are satisfied without need for computation. Systematic $h$-astral configurations are those where $S \neq T$, but the values of $S$ and $T$ are determined by dependence on distinct parameters. Sporadic $h$-astral configurations are neither trivial nor systematic.

\section{Constructing symmetric $(4,6)$-configurations}

Consider the trivial 4-astral configuration shown in Figure 4. This configuration consists of four symmetry classes of points and four symmetry classes of lines, with four points, two of each of two colors, on each line. However, there are extra four-valent intersections formed 
by the intersection of some of the lines: specifically, the blue and green lines (lines $L_{0}$ and $L_{2}$ ) and the red and magenta lines (lines $L_{1}$ and $L_{3}$ ) intersect two at a time. Adding these additional points would form a (4,6)-configuration: each point would still have four lines passing through it, but each line would now have six points, from each of three symmetry classes, lying on it.

We can further analyze this example by realizing that the $(4,6)$-configuration thus formed may be considered as being constructed from two separate 4-astral configurations, which have the same sets of lines (although different labels), and the same points $v_{0}$ and $v_{2}$, but different points $v_{1}$ and $v_{3}$. Figure 5 shows such a situation: here the points $v_{0}$ and $v_{3}$, colored blue and green respectively, are the same in both configurations, and as sets the lines of the two configurations are the same as well, although the colors are switched.

Definition 3.1. Two $h$-astral configurations $\mathcal{X}$ and $\mathcal{Y}$ are superimposable if they have the same sets of lines, geometrically, and the incidence structure formed from those lines and the collection of points from both configurations is a $(4,6)$-configuration.

The superimposibility of $9 \#(3,1 ; 4,2 ; 1,3 ; 2,4)$ and $9 \#(3,2 ; 4,1 ; 2,3 ; 1,4)$ is not coincidental: in fact, there are infinitely many such pairs of superimposable configurations.

To prove this, we will show that a particular pair of configurations $\mathcal{X}$ and $\mathcal{Y}$ is superimposable, by showing that they have the same set of lines and that the points $\left(v_{\mathcal{X}}\right)_{0}=\left(v_{\mathcal{Y}}\right)_{0}$ and $\left(v_{\mathcal{X}}\right)_{2}=\left(v_{\mathcal{Y}}\right)_{2}$. To do this, we will need the following lemma, slightly restated from [6, Lemma 1]:

Lemma 3.2. For a given $i$, if $s_{i} \equiv t_{i} \bmod 2$, the points labelled $v_{i}$, with symbol $\left(s_{i} / / t_{i}\right)$, are the same type as the points labelled $v_{i-1}$ (that is, $\left(s_{i-1} / / t_{i-1}\right)$ ), with indices taken modulo $h$; if $s_{i} \not \equiv t_{i} \bmod 2$, then the points $v_{i}$ and $v_{i-1}$ are of opposite type.

Theorem 3.3. Let $\mathcal{X}$ and $\mathcal{Y}$ be valid configurations with symbols $\mathcal{X}=m \#\left(a, x_{1} ; x_{2}, d\right.$; $\left.b, x_{3} ; x_{4}, c\right)$ and $\mathcal{Y}=m \#\left(a, y_{1} ; y_{2}, b ; d, y_{3} ; y_{4}, c\right)$. If

$$
\frac{\cos \left(x_{2} \pi / m\right)}{\cos \left(x_{1} \pi / m\right) \cos (d \pi / m)}=\frac{\cos \left(y_{2} \pi / m\right)}{\cos \left(y_{1} \pi / m\right) \cos (b \pi / m)}
$$

and

$$
x_{1}+x_{2}+y_{1}+y_{2}+d+b \text { is even, }
$$

then $\mathcal{X}$ and $\mathcal{Y}$ are superimposable.

Proof. Suppose that $x_{1}+x_{2}+y_{1}+y_{2}+d+b$ is even and

$$
\frac{\cos \left(x_{2} \pi / m\right)}{\cos \left(x_{1} \pi / m\right) \cos (d \pi / m)}=\frac{\cos \left(y_{2} \pi / m\right)}{\cos \left(y_{1} \pi / m\right) \cos (b \pi / m)} .
$$

Let $\left(v_{\mathcal{X}}\right)_{0}=\left(v_{\mathcal{Y}}\right)_{0}=v_{0}$ be the set of points with coordinates

$$
\left(\cos \left(\frac{2 \pi i}{m}\right), \sin \left(\frac{2 \pi i}{m}\right)\right)
$$

for $i=0,1, \ldots, m-1$. By the choice of symbol, $\left(v_{\mathcal{X}}\right)_{0}$ and $\left(v_{\mathcal{Y}}\right)_{0}$ have lines of the same spans passing through them: that is, $\left(L_{\mathcal{X}}\right)_{0}=\left(L_{\mathcal{Y}}\right)_{0}$ and $\left(L_{\mathcal{X}}\right)_{3}=\left(L_{\mathcal{Y}}\right)_{3}$, which are lines of spans $a$ and $c$, respectively, with respect to the points $v_{0}$. 

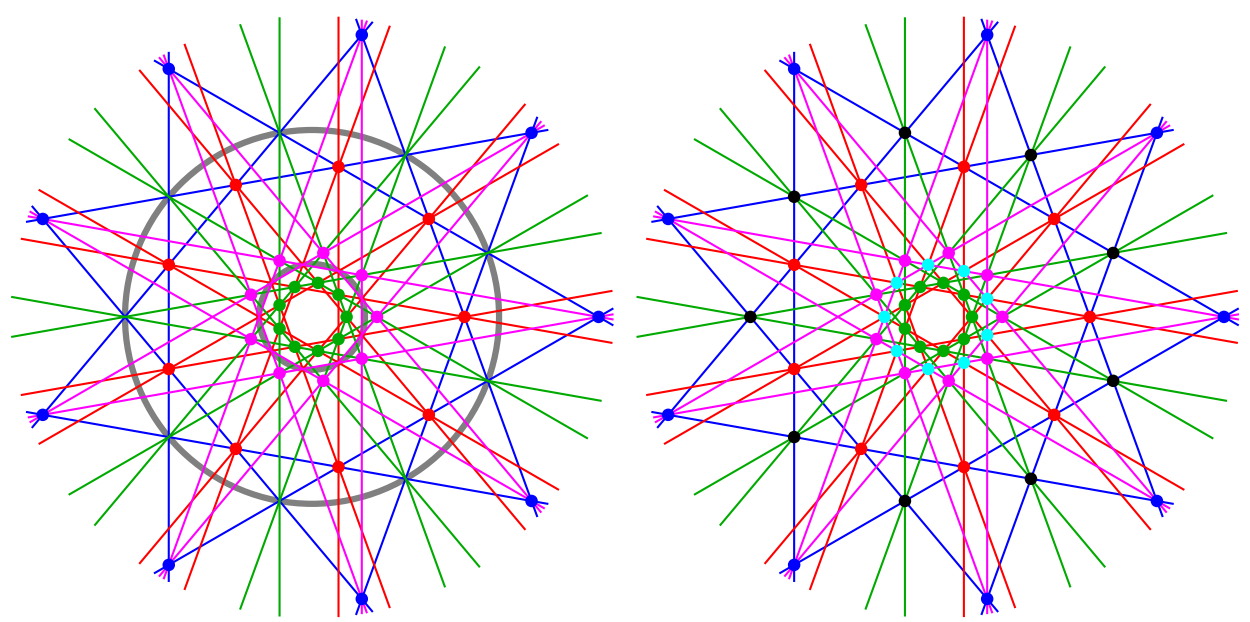

Figure 4: LHS: The trivial 4 -astral $\left(54_{4}, 36_{6}\right)$ configuration $9 \#(3,1 ; 4,2 ; 1,3 ; 2,4)$. There are additional four-valent intersections between the lines, specifically blue-green and redmagenta intersections, which are not points of the configuration; these are highlighted by the gray circles. RHS: Adding in the additional intersection points leads to a $(4,6)$ configuration; the additional points are black and cyan. With different coloring, this configuration is shown as Figure 4.4.10 in [12].
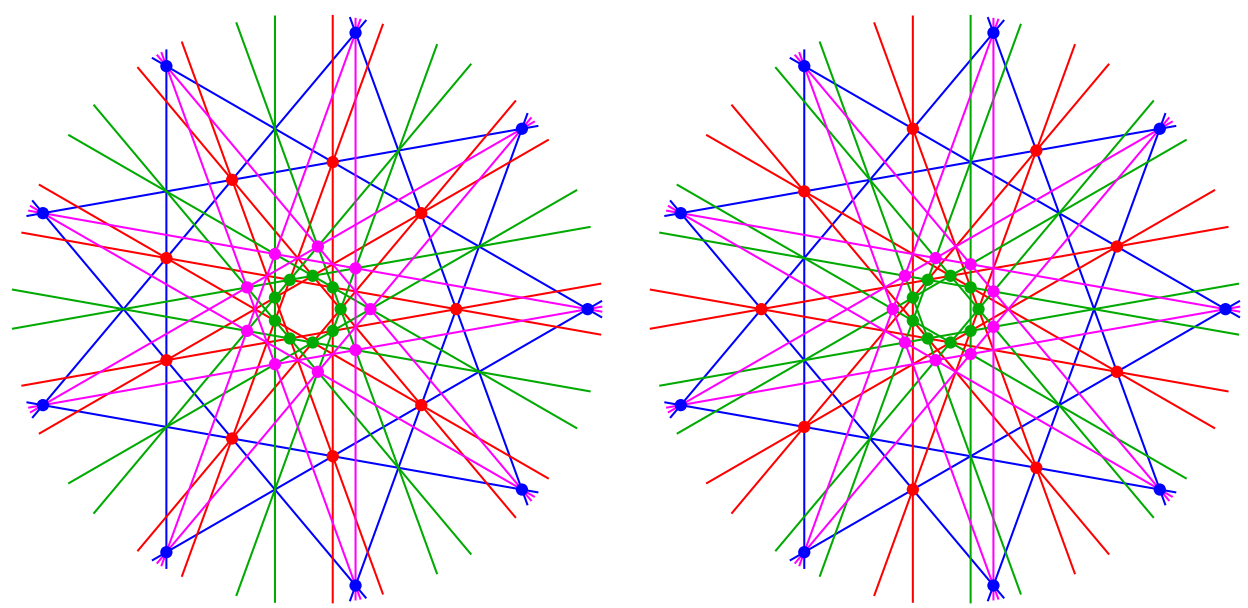

Figure 5: The two superimposable configurations which when combined form the $(4,6)$ configuration shown in the right-hand side of Figure 4. LHS: $9 \#(3,1 ; 4,2 ; 1,3 ; 2,4)$; RHS: $9 \#(3,2 ; 4,1 ; 2,3 ; 1,4)$. 


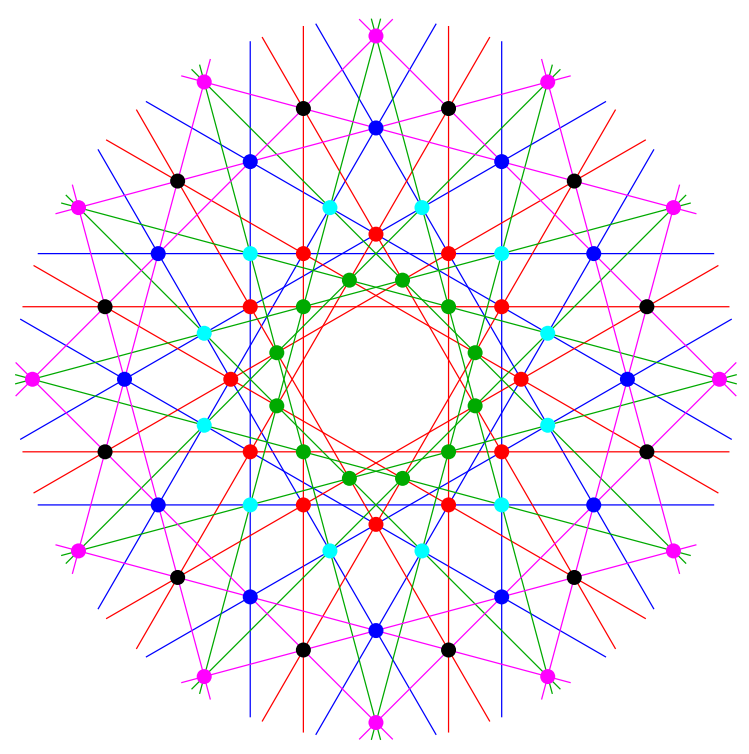

Figure 6: A $(4,6)$-configuration formed from the nontrivial superimposable pair $12 \#(4,2 ; 4,3 ; 2,5 ; 3,1)$ and $12 \#(4,3 ; 4,2 ; 3,5 ; 2,1)$.

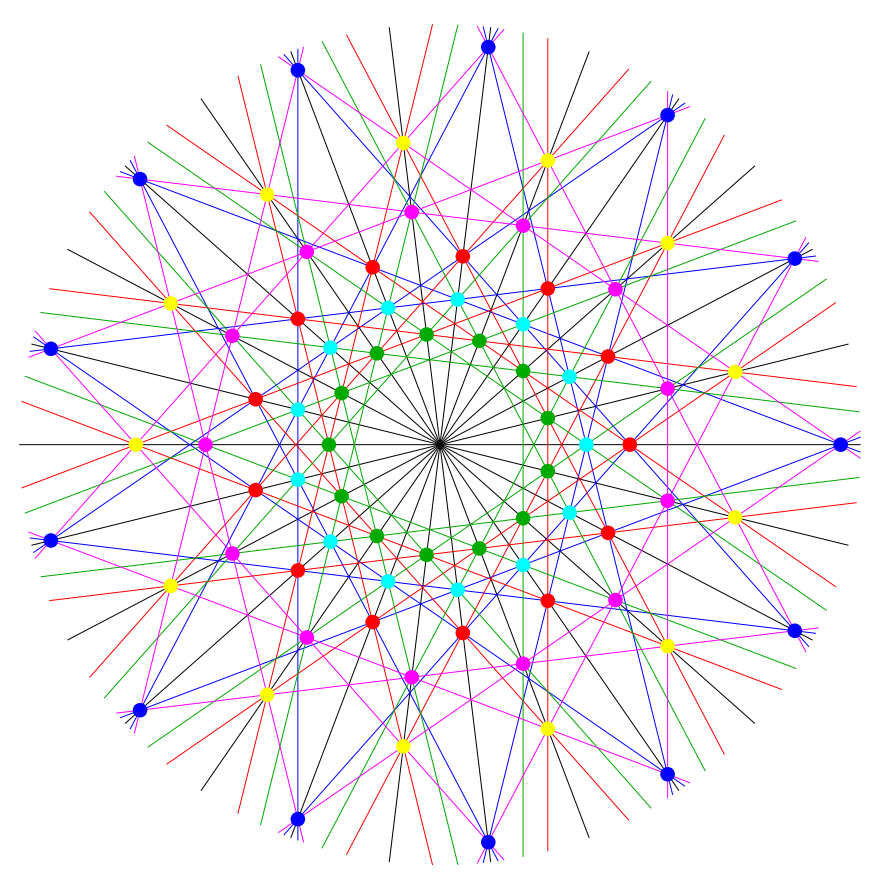

Figure 7: A $(5,6)$-configuration formed by adding diameters to the superposition of $13 \#(5,3 ; 4,1 ; 3,5 ; 1,4)$ (blue, red, green, and magenta points) and $13 \#(5,1 ; 4,3 ; 1,5 ; 3,4)$ (blue, yellow, green, and cyan points). 
Note that, again by the choice of symbol, the points $\left(v_{\mathcal{X}}\right)_{2}$ have lines $\left(L_{\mathcal{X}}\right)_{2}$ of span $d$ and $\left(L_{\mathcal{X}}\right)_{1}$ of span $c$ passing through them; similarly, the points $\left(v_{\mathcal{Y}}\right)_{2}$ have lines $\left(L_{\mathcal{Y}}\right)_{2}$ of span $d$ and $\left(L_{\mathcal{Y}}\right)_{1}$ of span $c$ passing through them. Therefore, to show that $\mathcal{X}$ and $\mathcal{Y}$ are superimposable, it suffices to show that $\left(v_{\mathcal{X}}\right)_{2}$ is the same set of points, geometrically, as $\left(v_{\mathcal{Y}}\right)_{2}$, which we will do by showing that they are of the same type and have the same radius.

Lemma 3.2 states that $v_{i-1}$ and $v_{i}$ are of the same type if $s_{i}$ and $t_{i}$ have the same parity; that is, when $s_{i}+t_{i}$ is even. Following the type changes through the symbol, if $\left(v_{\mathcal{X}}\right)_{2}$ and $\left(v_{y}\right)_{2}$ are of the same type, then $a+x_{1}+x_{2}+d$ and $a+y_{1}+y_{2}+b$ must have the same parity, since $\left(v_{\mathcal{X}}\right)_{0}=\left(v_{\mathcal{Y}}\right)_{0}$. Since we assumed that $x_{1}+x_{2}+y_{1}+y_{2}+d+b$ is even, it follows that $\left(v_{\mathcal{X}}\right)_{2}$ and $\left(v_{\mathcal{Y}}\right)_{2}$ are of the same type.

Now, let $\left(r_{\mathcal{X}}\right)_{i}$ and $\left(r_{\mathcal{Y}}\right)_{i}$ be the radii of $\left(v_{\mathcal{X}}\right)_{i}$ and $\left(v_{\mathcal{Y}}\right)_{i}$ respectively. By construction, $\left(r_{\mathcal{X}}\right)_{0}=\left(r_{\mathcal{Y}}\right)_{0}=1$. Then

$$
\left(r_{\mathcal{X}}\right)_{2}=\left(r_{\mathcal{X}}\right)_{1} \cdot \frac{\cos \left(x_{2} \pi / m\right)}{\cos (d \pi / m)}=\frac{\cos (a \pi / m) \cos \left(x_{2} \pi / m\right)}{\cos \left(x_{1} \pi / m\right) \cos (d \pi / m)}
$$

and

$$
\left(r_{\mathcal{Y}}\right)_{2}=\left(r_{\mathcal{Y}}\right)_{1} \cdot \frac{\cos \left(y_{2} \pi / m\right)}{\cos (b \pi / m)}=\frac{\cos (a \pi / m) \cos \left(y_{2} \pi / m\right)}{\cos \left(y_{1} \pi / m\right) \cos (b \pi / m)} .
$$

Since we assumed that

$$
\frac{\cos \left(x_{2} \pi / m\right)}{\cos \left(x_{1} \pi / m\right) \cos (d \pi / m)}=\frac{\cos \left(y_{2} \pi / m\right)}{\cos \left(y_{1} \pi / m\right) \cos (b \pi / m)},
$$

it follows that $\left(r_{\mathcal{X}}\right)_{2}=\left(r_{\mathcal{Y}}\right)_{2}$, as desired.

Corollary 3.4. Superimposable configurations

$$
\mathcal{X}=m \#\left(a, x_{1} ; x_{2}, d ; b, x_{3} ; x_{4}, c\right)
$$

and

$$
\mathcal{Y}=m \#\left(a, y_{1} ; y_{2}, b ; d, y_{3} ; y_{4}, c\right)
$$

satisfy

$$
\frac{\cos (b \pi / m) \cos \left(x_{4} \pi / m\right)}{\cos \left(x_{3} \pi / m\right)}=\frac{\cos (d \pi / m) \cos \left(y_{4} \pi / m\right)}{\cos \left(y_{3} \pi / m\right)} .
$$

Proof. Since $\mathcal{X}$ and $\mathcal{Y}$ are both valid configurations, the radius $r_{4}$ for each configuration must equal 1 , because $v_{4}=v_{0}$. Since $\mathcal{X}$ and $\mathcal{Y}$ are superimposable, $\left(v_{\mathcal{X}}\right)_{2} \equiv\left(v_{\mathcal{Y}}\right)_{2}$; call the common radius $r$. Then

$$
\left(r_{\mathcal{X}}\right)_{4}=r \cdot \frac{\cos \left(\frac{b \pi}{m}\right)}{\cos \left(\frac{x_{3} \pi}{m}\right)} \cdot \frac{\cos \left(\frac{x_{4} \pi}{m}\right)}{\cos \left(\frac{c \pi}{m}\right)}
$$

and

$$
\left(r_{\mathcal{Y}}\right)_{4}=r \cdot \frac{\cos \left(\frac{d \pi}{m}\right)}{\cos \left(\frac{y_{3} \pi}{m}\right)} \cdot \frac{\cos \left(\frac{y_{4} \pi}{m}\right)}{\cos \left(\frac{c \pi}{m}\right)} .
$$

Since $\left(r_{\mathcal{X}}\right)_{4}=\left(r_{\mathcal{Y}}\right)_{4}=1$, the result follows. 
One very nice class of pairs of superimposable configurations are the trivial pairs

$$
\mathcal{X}=m \#(a, b ; c, d ; b, a ; d, c) \quad \text { and } \quad \mathcal{Y}=m \#(a, d ; c, b ; d, a ; b, c)
$$

these trivially satisfy Theorem 3.3. Figure 5 shows such a trivial pair. Figure 6 shows a $(4,6)$-configuration formed from the nontrivial pair $12 \#(4,2 ; 4,3 ; 2,5 ; 3,1)$ and $12 \#(4,3$; $4,2 ; 3,5 ; 2,1)$.

\section{Generalizations and open questions}

Given a $(4,6)$-configuration with three symmetry classes of points of one type and three of the other type, it is possible to construct symmetric $(5,6)$-configurations by adding diameters; an example of such a configuration is shown in Figure 7.

In particular, consider a superimposable pair of trivial configurations $\mathcal{X}=m \#(a, b$; $c, d ; b, a ; d, c)$ and $\mathcal{Y}=m \#(a, d ; c, b ; d, a ; b, c)$. Suppose that $a, b, d$ are of the same parity and $c$ is of the opposite parity to $a, b, d$. Applying Lemma 3.2 several times, we conclude that $\left(v_{\mathcal{X}}\right)_{0}=\left(v_{\mathcal{Y}}\right)_{0}$ are type $D,\left(v_{\mathcal{X}}\right)_{1}$ and $\left(v_{\mathcal{Y}}\right)_{1}$ are type $D$, since $a \equiv b \equiv d \bmod 2$, $\left(v_{\mathcal{X}}\right)_{2}=\left(v_{\mathcal{Y}}\right)_{2}$ is of type $M D$, since $c \not \equiv b \bmod 2$ and $c \not \equiv d \bmod 2$, and $\left(v_{\mathcal{X}}\right)_{3}$ and $\left(v_{\mathcal{Y}}\right)_{3}$ are both of type $M D$, since $a \equiv b \equiv d \bmod 2$. Thus, in the superimposed $(4,6)$ configuration, there are three classes of points of type $D$, namely $\left(v_{\mathcal{X}}\right)_{0},\left(v_{\mathcal{X}}\right)_{1}$ and $\left(v_{\mathcal{Y}}\right)_{1}$, and three classes of points of type $M D,\left(v_{\mathcal{X}}\right)_{2},\left(v_{\mathcal{X}}\right)_{3}$, and $\left(v_{\mathcal{Y}}\right)_{3}$, so if diameters (that is, lines connecting the origin and points in $\left.v_{0}\right)$ are added to the configuration, each diameter will pass through six points.

There are several interesting ways to generalize the notion of superimposibility. For example, consider two configurations to be superimposable if they

- share the same point sets, but different line sets, but combine into some $(q, k)$ configuration

- Have different point and line sets, but still combine to form a $(q, k)$-configuration

Clearly, the $(6,4)$-configurations formed as the polars of the $(4,6)$-configurations constructed above may be analyzed as being formed from two superimposable 4-astral configurations which share the same point sets but different line sets.

Question 1. A 4-astral configuration cohort $m \# S ; T$ is reducible if $S=\left\{x_{1}, x_{2}, i, j\right\}$ and $T=\left\{y_{1}, y_{2}, i, j\right\}$ and $m \# S^{\prime} ; T^{\prime}$ is a valid 2-astral configuration, where $S^{\prime}=\left\{x_{1}, x_{2}\right\}$ and $T^{\prime}=\left\{y_{1}, y_{2}\right\}$. So far, the only known nontrivial superimposable pairs are reducible (for example, the configurations which superimpose to form the $(4,6)$-configuration in Figure 6 both reduce to the configuration cohort $12 \#\{4,4\} ;\{5,1\}$.) Are there nonreducible pairs of superimposable 4-astral configurations?

Question 2. Are there other interesting configurations which can be formed by superposition, perhaps using $h$-astral configurations for $h>4$ ?

Question 3. Is it possible to construct interesting configurations by superimposing more than two configurations?

Question 4. Is it possible to construct 6-configurations by superimposing multiple $(4,6)$ or $(6,4)$-configurations? 


\section{Acknowledgements}

The authors are grateful to the Ursinus College Summer Fellows program for providing support for this research.

\section{References}

[1] L. W. Berman, A characterization of astral $\left(n_{4}\right)$ configurations, Disc. Comp. Geom. 26 (2001), 603-612.

[2] L. W. Berman, Even astral configurations, Electron. J. Combin. 11 (2004), \#R37.

[3] L. W. Berman, Some results on odd astral configurations. Electron. J. Combin. 13 (2006), \#R27.

[4] L. W. Berman, Movable $\left(n_{4}\right)$ Configurations, Electron. J. Combin. 13 (2006), \#R104.

[5] L. W. Berman, J. Bokowski, B. Grünbaum and T. Pisanski. Geometric "floral" configurations, Canadian Math. Bull. 52 (2009), 327-341.

[6] L. W. Berman and B. Grünbaum, Deletion Constructions of Symmetric 4-Configurations, Part I, Contrib. Disc. Math. 5 (2010).

[7] M. Boben and T. Pisanski, Polycyclic configurations, European J. Combin. 24 (2003), 431457.

[8] J. Bokowski, B. Grünbaum and L. Schewe, Topological configurations $\left(n_{4}\right)$ exist for all $n \geq 17$, European J. Combin. 30 (2009), 1778-1785.

[9] B. Grünbaum, Astral ( $\left.n_{k}\right)$ configurations, Geombinatorics 3 (1993), 32-37.

[10] B. Grünbaum, Astral $\left(n_{4}\right)$ configurations, Geombinatorics 9 (2000), 127-134.

[11] B. Grünbaum, Configurations of points and lines, in C. Davis and E. W. Ellers (eds.), The Coxeter Legacy, Reflections and Projections, American Mathematical Society (2006), 179225.

[12] B. Grünbaum, Configurations of points and lines, American Mathematical Society, 2009.

[13] B. Grünbaum and J. F. Rigby, The real configuration (214), J. London Math. Soc (2) 41 (1990), 336-346. 